Letter

\title{
The Promyelocytic Leukemia Protein Is Upregulated in Conditions of Obesity and Liver Steatosis
}

\author{
Arkaitz Carracedo $1,2,3,4,{ }^{*}$, , Déborah Rousseau ${ }^{5,6^{*}}$, Nicholas Douris ${ }^{7^{*}}$, Sonia Fernández-Ruiz ${ }^{2}$, Natalia \\ Martín-Martín' ${ }^{2}$, Dror Weiss ${ }^{1}$, Kaitlyn Webster ${ }^{1}$, Andrew C. Adams 7,8 , Mercedes Vazquez-Chantada ${ }^{2}$, Maria \\ L. Martinez-Chantar2,9, Rodolphe Anty5,6,10, Albert Tran5,6,10, Eleftheria Maratos-Flier7, Philippe Gual5,6, Pier \\ Paolo Pandolfi ${ }^{1, \bigotimes}$ \\ 1. Cancer Research Institute, Beth Israel Deaconess Cancer Center, Department of Medicine and Pathology, Beth Israel Deaconess Medical Center, \\ Harvard Medical School, Boston, MA, USA \\ 2. CIC bioGUNE, Bizkaia Technology Park, Derio, Spain \\ 3. IKERBASQUE, Basque foundation for science, Bilbao, Spain \\ 4. Biochemistry and Molecular Biology Department, University of the Basque Country (UPV/EHU), P. O. Box 644 , E-48080 Bilbao, Spain \\ 5. INSERM, U1065, Team 8, "Hepatic Complications in Obesity", Nice, F-06204, Cedex 3, France \\ 6. University of Nice-Sophia-Antipolis, Faculty of Medecine, Nice, F-06107, Cedex 2, France \\ 7. Division of Endocrinology; Department of Medicine,Beth Israel Deaconess Medical Center, Harvard Medical School, Boston MA 02215, USA \\ 8. Lilly Research Laboratories, Lilly Corporate Center, Indianapolis, Indiana, USA \\ 9. Centro de Investigación Biomédica en Red de Enfermedades Hepáticas y Digestivas (Ciberehd) \\ 10. CHU of Nice, Digestive Center, Nice, F-06202, Cedex 3, France
}

${ }^{*}$ Equal contribution

$\triangle$ Corresponding authors: acarracedo@cicbiogune.es and ppandolf@bidmc.harvard.edu

() 2015 Ivyspring International Publisher. Reproduction is permitted for personal, noncommercial use, provided that the article is in whole, unmodified, and properly cited. See http://ivyspring.com/terms for terms and conditions.

Received: 2015.01.16; Accepted: 2015.02.24; Published: 2015.04.11

The promyelocytic leukemia protein (PML) is the essential component of multi-protein sub-nuclear structures, the PML-Nuclear bodies (PML-NB, [1]). The PML-NBs regulate a variety of nuclear functions, including post-translational modifications in nuclear proteins (acetylation, SUMOylation, ubiquitylation) [1]. This function has led to the current notion that PML is a modulator of cell responses, which might not be essential in steady-state conditions (under lack of stress) but plays a critical role when the cell and organism is challenged. This notion is supported by the fact that the lack of PML in mice does not result in embryonic lethality or overt physiological alterations in adulthood, but it alters the response to oncogenic insults or dietary alterations [1-4].

The activity of PML has been mostly investigated in the context of suppression, pathogenesis and progression of tumorigenesis. However, evidence of the function of this protein beyond malignant transformation are quickly accumulating. We and others have recently reported that the expression of PML is relevant for the response to metabolic insults, nutritional disorders and obesity [4-8]. In this study, we aimed at extending and defining the status of PML in conditions of nutritional challenge. To this end, we evaluated PML transcript abundance in a cohort of human liver biopsies from lean or morbidly obese subjects. Liver material from lean patients was obtained from 3 subjects ( 3 women; age, $46 \pm 12$ years; BMI, $21 \pm 2 \mathrm{~kg} / \mathrm{m}^{2}$ ) undergoing partial hepatectomy for benign tumors (neighbor tissues from four adenoma and one focal nodular hyperplasia) and did not display any hepatic steatosis, inflammation or fibrosis. For obese patients, bariatric surgery was indicated in accordance with French guidelines (the study was approved by "Comité Consultatif de Protection des Personnes dans la Recherche Biomédicale de Nice" 07/04:2003, $N^{\circ}$ 03.017) (Fig. 1A). Quantitative real time RT-PCR (q-RT-PCR) analysis (references from Applied biosystems Hs99999902_m1 for housekeeping RPLPO (Ribosomal protein, large subunit, P0) and Hs00231241_m1 for PML and as previously described [9, 10]), revealed a significant PML up-regulation in obese individuals (Fig. 1B). Further, hepatic PML expression increased in obese patients without liver complications $(n=5)$ versus lean subjects $(+2.49 \pm 0.28$, $\mathrm{P}=0.036$ ). We next decided to extend this observation to diet-induced (Research Diets D12451 as obesogenic 
diet; LabDiet 5008 (Pharmaserv, Framingham, MA, USA) as chow, following the procedure in Ref. [11]) or genetic (Agouti mutant heterozygous) mouse models of obesity (the mouse experiments were approved by IACUC, USA). Q-RT-PCR analysis in high-fat diet fed mice demonstrated the up-regulation of Pml in livers from obese mice (murine Pml primers, Pml_F: GATCTCCGCGACAATTCAGT, Pml_R: ATGCCACT GCTGAATCTCCT; Cyclophilin was used as housekeeping gene; Cyclo_F: GGTGGAGAGCACCAAG ACAGA, Cyclo_R: GCCGGAGTCGACAATGATG; Fig. 1C). Importantly, transcriptional up-regulation of this gene was accompanied by the detection of Pml A

B immunoreactive nuclear bodies by immunohistochemistry in $<5 \%$ of hepatocytes in livers from HFD mice (using Millipore Pml antibody), but rarely in those from lean individuals (Fig. 1D), similar to our prior observations in another mouse model [4]. Of note, Pml immunoreactivity was often restricted to a single or few large nuclear bodies of doughnut shape, a structural conformation prior described for PML nuclear bodies. We also confirmed the increase Pml immunoreactivity in a second murine model of obesity, driven by the heterozygous mutation in the Agouti gene (Jackson laboratory ref. KK.Cg- $A^{y} / \mathrm{J}$; Ref. [12]; Fig. 1E).

\section{C}

\begin{tabular}{|c|c|c|c|}
\hline & \multicolumn{3}{|c|}{ Liver } \\
\hline $\mathrm{n}(\mathrm{F} / \mathrm{M})$ & \multicolumn{3}{|c|}{$23(21 / 2)$} \\
\hline Age (years) & 38 & \pm & 2 \\
\hline BMI $\left(\mathrm{kg} / \mathrm{m}^{2}\right)$ & 43 & \pm & 1 \\
\hline T2D (n) & \multicolumn{3}{|c|}{4} \\
\hline Fasting insulin (mIU/L) & 14.2 & \pm & 2.6 \\
\hline Fasting glucose (mmol/L) & 6.2 & \pm & 0.6 \\
\hline HOMA-IR & 4.7 & \pm & 1.4 \\
\hline HbA1c (\%) & 6.2 & \pm & 0.4 \\
\hline Triglycerides (mmol/L) & 1.4 & \pm & 0.1 \\
\hline HDL cholesterol (mmol/L) & 1.4 & \pm & 0.1 \\
\hline LDL cholesterol (mmol/L) & 3.1 & \pm & 0.1 \\
\hline Without NAFLD (n) & \multicolumn{3}{|c|}{5} \\
\hline With Steatosis (n) & \multicolumn{3}{|c|}{18} \\
\hline $\mathrm{S1}(\mathrm{n})$ & \multicolumn{3}{|c|}{6} \\
\hline S2 (n) & \multicolumn{3}{|c|}{4} \\
\hline S3 (n) & \multicolumn{3}{|c|}{8} \\
\hline ALT (IU/L) & 29.3 & \pm & 5.0 \\
\hline
\end{tabular}

PML gene expression

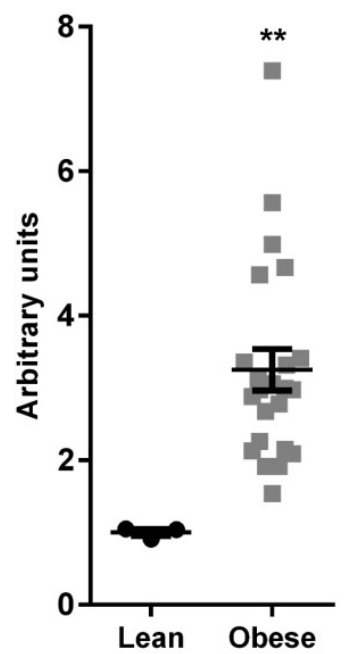

Pml gene expression

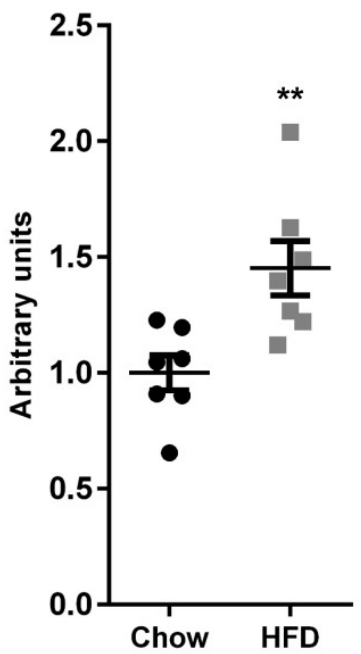

D

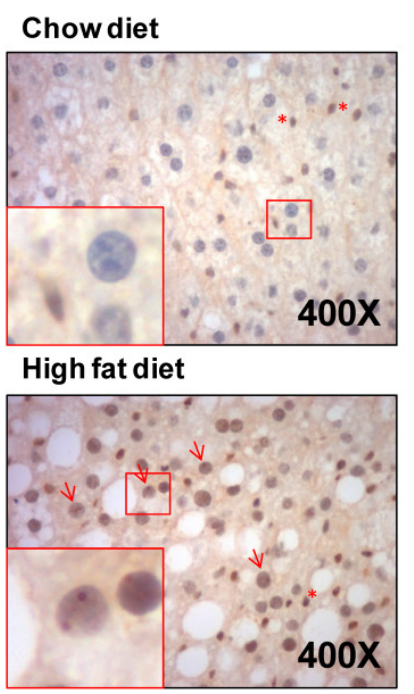

$\mathbf{E}$
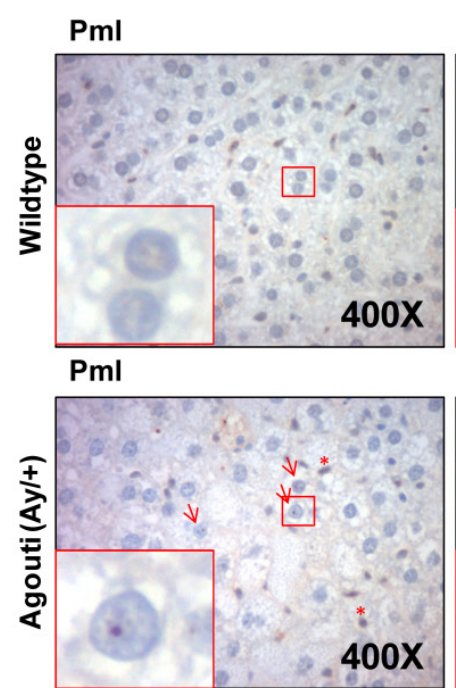

Sumo-1

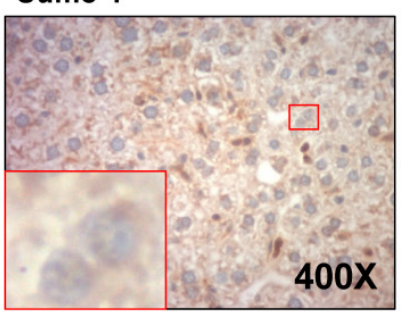

Sumo-1

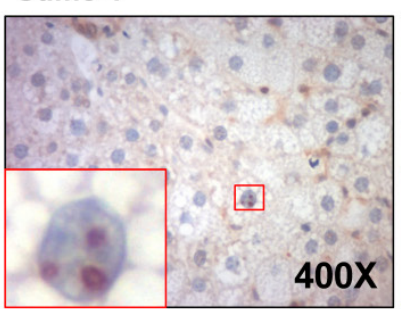

Npm

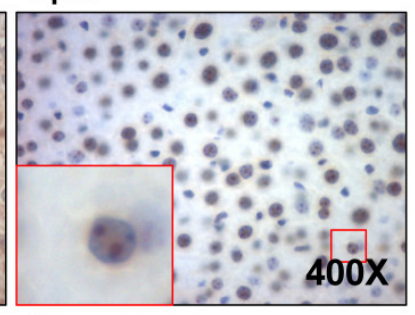

Npm

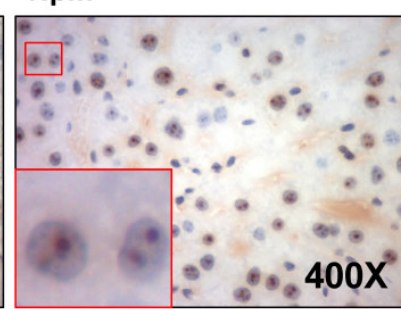

Figure 1.PML is up-regulated in hepatocytes from obese individuals. (A-B) Description of the clinical features of obese subjects (A) in which PML transcript abundance (q-RT-PCR) was measured (B). (C-D). Pml transcript abundance (q-RT-PCR) (C) and immunoreactivity (D) in mice subjected to standard (chow) or obesity-inducing high-fat diets (HFD) (representative micrographs from $n=4-5$; squares represent zoomed region; red arrows depict PML immunoreactive hepatocytes; red asterisks depict PML immunoreactive non-hepatocyte cells). $* *, p<0.01$ calculated by non-parametric Mann-Whitney test. (E) Pml, Sumo-1 and Nucleophosmin $(\mathrm{Npm})$ immunoreactivity in livers from wild type or agouti heterozygous mutant mice (representative micrographs from $n=3$; squares, arrows and asterisks as described in D). 
PML nuclear bodies are enriched in SUMO-1 (Santa Cruz BioTechnology). Immunohistochemical analysis confirmed similar nuclear distribution of Sumo-1 into nuclear bodies at a similar rate of Pml, which strongly suggests that the staining of Pml corresponds to functional nuclear bodies (Fig. 1E). It is worth noting that this altered pattern of nuclear localization was not observed in other non PML-NB resident proteins such as nucleophosmin (a nucleolar protein; Cell Signaling Technology; Fig. 1E). In summary, our results demonstrate that PML accumulates in hepatocytes in conditions of obesity.

Obesity is associated to lipid accumulation in hepatocytes, termed steatosis [13]. Since there are pathological conditions where steatosis can be triggered in the absence of obesity, we sought to ascertain whether PML accumulation was related to obesity or it was a consequence of the steatosis associated to weight increase. To this end, we first evaluated PML expression in obese patients with different degree of steatosis $(0,<5 \% ; 1,5 \%-30 \% ; 2,>30 \%-60 \% ; 3,>60 \%)$ [14]. The results clearly demonstrated that PML expression significantly correlated with the extent of steatosis (Fig. 2A).

A

B
In addition, we took advantage of a dietary regime that results in weight loss and liver lipid accumulation, namely ketogenic diet (KD; Ref. Bio-Serv F3666, [11]). The results confirmed that Pml transcript and immunoreactivity were increased (Fig. 2B-C).

Fatty liver disease is a rapidly increasing pathology that, albeit tightly associated with obesity, is also observed in a fraction of lean individuals, where it is thought to be related to nutrition and life style factors [13]. Our results conclusively demonstrate that PML expression is increased in livers from obese individuals, and is particularly associated to a steatotic phenotype. These findings are of relevance for the characterization of PML non-tumoral functions. Importantly, this observation might open an interesting mean of regulation of PML in the context of cancerous lesions, in which nutrient availability might impact on the expression of this protein and hence on the biology of the disease. In particular, they extend our knowledge regarding the patho-physiological regulation of PML in metabolism. In this respect, it is tempting to speculate that PML induction may represent a failsafe response to steatosis in view of the positive role it plays in the activation fatty acid oxidation pathways $[4,5]$.
PML gene expression

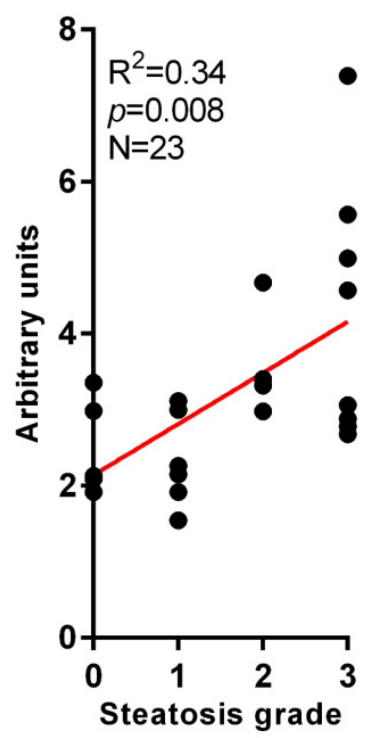

Pml gene expression

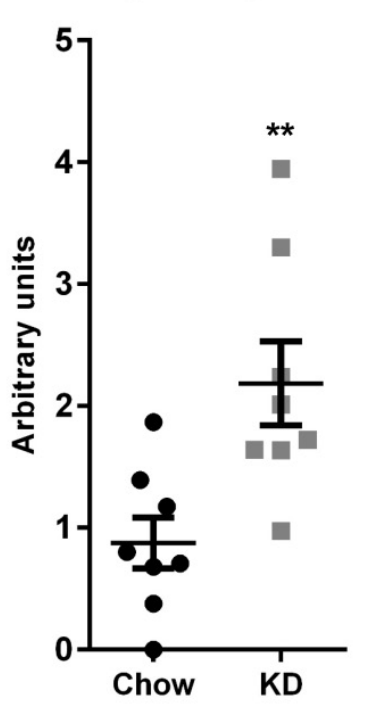

C 
Cancer (AECC). The work of P.G. was supported by grants from INSERM (France), the University of Nice, the Programme Hospitalier de Recherche Clinique (Centre Hospitalier Universitaire of Nice), and charities (Association Française pour l'Etude du Foie, Société Francophone du Diabète and SFD/Roche Pharma, European Foundation for the study of Diabetes/Lilly European Diabetes Research Program and the French Government (National Research Agency, ANR) through the "Investments for the Future" (LABEX SIGNALIFE: program reference \#ANR-11-LABX-0028-01). The work of P.P.P. and A.C. was supported by the National Institutes of Health (NIH, USA) grants. N.D. was supported by NRSA 5T32DK751627 (NIH, USA). M.L.M.-C was funded by Sanidad Gobierno Vasco 2013, FIS PI11/01588.

\section{Competing Interests}

The authors declare no competing financial interest.

\section{References}

1. Bernardi R, Pandolfi PP. Structure, dynamics and functions of promyelocytic leukaemia nuclear bodies. Nat Rev Mol Cell Biol. 2007; 8: 1006-16. doi:nrm2277 [pii]10.1038/nrm2277.

2. Bernardi R, Pandolfi PP. Role of PML and the PML-nuclear body in the control of programmed cell death. Oncogene. 2003; 22: 9048-57. doi:10.1038/sj.onc.12071061207106 [pii].

3. Carracedo A, Ito K, Pandolfi PP. The nuclear bodies inside out: PML conquers the cytoplasm. Curr Opin Cell Biol. 2011; 23: 360-6. doi:S0955-0674(11)00028-7 [pii]10.1016/j.ceb.2011.03.011.

4. Carracedo A, Weiss D, Leliaert AK, Bhasin M, de Boer VC, Laurent G, et al. A metabolic prosurvival role for PML in breast cancer. The Journal of clinical investigation. 2012; 122: 3088-100. doi:62129 [pii]10.1172/JCI62129.

5. Ito K, Carracedo A, Weiss D, Arai F, Ala U, Avigan DE, et al. A PML-PPAR- $\delta$ pathway for fatty acid oxidation regulates hematopoietic stem cell maintenance. Nat Med. 2012; 18: 1350-8. doi:nm.2882 [pii]10.1038/nm.2882.

6. Kim MK, Yang S, Lee KH, Um JH, Liu M, Kang H, et al. Promyelocytic Leukemia (PML) Inhibits Adipogenesis and Loss of PML Results in Fat Accumulation in Mice. Am J Physiol Endocrinol Metab. 2011 Dec;301(6):E1130-42..

7. Cheng X, Guo S, Liu Y, Chu H, Hakimi P, Berger NA, et al. Ablation of promyelocytic leukemia protein (PML) re-patterns energy balance and protects mice from obesity induced by a Western diet. J Biol Chem. 2013; 288: 29746-59. doi:10.1074/jbc.M113.487595.

8. Kitamura YI, Kitamura T, Kruse JP, Raum JC, Stein R, Gu W, et al. FoxO1 protects against pancreatic beta cell failure through NeuroD and MafA induction. Cell Metab. 2005; 2: 153-63. doi:S1550-4131(05)00232-9 [pii]10.1016/j.cmet.2005.08.004.

9. Anty R, Bekri S, Luciani N, Saint-Paul MC, Dahman M, Iannelli A, et al. The inflammatory C-reactive protein is increased in both liver and adipose tissue in severely obese patients independently from metabolic syndrome, Type 2 diabetes, and NASH. Am J Gastroenterol. 2006; 101: 1824-33. doi:10.1111/j.1572-0241.2006.00724.x.

10. Bekri S, Gual P, Anty R, Luciani N, Dahman M, Ramesh B, et al. Increased adipose tissue expression of hepcidin in severe obesity is independent from diabetes and NASH. Gastroenterology. 2006; 131: 788-96. doi:10.1053/j.gastro.2006.07.007.

11. Kennedy AR, Pissios P, Otu H, Roberson R, Xue B, Asakura K, et al. A high-fat, ketogenic diet induces a unique metabolic state in mice. Am J Physiol Endocrinol Metab. 2007; 292: E1724-39. doi:10.1152/ajpendo.00717.2006.

12. Bultman SJ, Michaud EJ, Woychik RP. Molecular characterization of the mouse agouti locus. Cell. 1992; 71: 1195-204.

13. Fracanzani AL, Valenti L, Bugianesi E, Vanni E, Grieco A, Miele L, et al. Risk of nonalcoholic steatohepatitis and fibrosis in patients with nonalcoholic fatty liver disease and low visceral adiposity. J Hepatol. 2011; 54: 1244-9. doi:10.1016/j.jhep.2010.09.037.

14. Kleiner DE, Brunt EM, Van Natta M, Behling C, Contos MJ, Cummings OW, et al. Design and validation of a histological scoring system for nonalcoholic fatty liver disease. Hepatology. 2005; 41: 1313-21. doi:10.1002/hep.20701. 